\title{
Formaldehyde sensor using non-dispersive UV spectroscopy at 340nm
}

\author{
J. J. Davenport ${ }^{\text {a }}$, J. Hodgkinson ${ }^{* a}$, J. R. Saffell ${ }^{b}$ and R. P. Tatam ${ }^{\text {a }}$ \\ a: Engineering Photonics, School of Engineering, Cranfield University, Bedfordshire, MK43 OAL, UK \\ b: Alphasense Ltd, Sensor Technology House, 300 Avenue West, Skyline 120, Great Notley, Essex CM77 7AA, UK
}

\begin{abstract}
Formaldehyde is a volatile organic compound that exists as a gas at room temperature. It is hazardous to human health causing irritation of the eyes, nose and throat, headaches, limited pulmonary function and is a potential human carcinogen. Sources include incomplete combustion, numerous modern building materials and vehicle fumes. Here we describe a simple method for detecting formaldehyde using low resolution non-dispersive UV absorption spectroscopy for the first time. A two channel system has been developed, making use of a strong absorption peak at $339 \mathrm{~nm}$ and a neighbouring region of negligible absorption at $336 \mathrm{~nm}$ as a reference. Using a modulated UV LED as a light source and narrowband filters to select the desired spectral bands, a simple detection system was constructed that was specifically targeted at formaldehyde. A minimum detectable absorbance of $4.5 \times 10^{-5} \mathrm{AU}$ was estimated (as $\left.\Delta \mathrm{I} / \mathrm{I}_{0}\right)$, corresponding to a limit of detection of approximately $6.6 \mathrm{ppm}$ for a $195 \mathrm{~mm}$ gas cell, with a response time of $20 \mathrm{~s}$. However, thermallyinduced drift in the LED spectral output caused this to deteriorate over longer time periods to around $30 \mathrm{ppm}$ or $2 \times 10^{-4} \mathrm{AU}$.
\end{abstract}

\section{Keywords: formaldehyde, gas sensing, UV spectroscopy, non-dispersive, NDUV}

\section{INTRODUCTION}

Formaldehyde $\left(\mathrm{CH}_{2} \mathrm{O}\right)$ is a colourless gas under standard conditions which is toxic, allergenic and a potential human carcinogen $^{[1,2,3,4]}$. The World Health Organisation (WHO) has set a guideline maximum level for prolonged or acute formaldehyde exposure at $80 \mathrm{ppb}$ (parts per billion), and many countries have set theirs in line with this ${ }^{[1,5]}$. However, formaldehyde is also a valuable industrial chemical with limited alternatives ${ }^{[1,4]}$. Formaldehyde based resin is used as an adhesive in plywood and medium density fibre board, in carpeting, and in the production of paints and wallpapers. It is also a by-product of incomplete combustion ${ }^{[1]}$ and is present in various cleaning products ${ }^{[1]}$. Many of these materials emit low concentrations of formaldehyde over time. Emission levels are highest when materials are new, generally decreasing exponentially, but can take multiple years to reach safe levels ${ }^{[1,5]}$. Formaldehyde gas can therefore build up in enclosed areas, particularly when new furnishings or carpeting have been installed and where premises are not adequately ventilated.

At the time of writing, sensors for formaldehyde that are compact and low cost are extremely limited. One electrochemical formaldehyde detector has been produced (PPM Technology Formaldemeter ${ }^{[6]}$. It has a response time of one minute and can measure formaldehyde concentrations at or below the WHO limit. However, electrochemical sensors generally suffer from limited lifetimes and their response can be compromised by cross-sensitivity to other species, including humidity.

High resolution optical techniques employing tunable diode laser spectroscopy (TDLS) have previously shown good sensitivity. For example, Rehle et al. ${ }^{[7]}$ reported formaldehyde detection at $0.32 \mathrm{ppb}$ using a $353 \mathrm{~nm}$ laser, and Richter $e t$ al. ${ }^{[8]}$ achieved a limit of detection of $74 \mathrm{ppt}$ at $3.5 \mu \mathrm{m}$, but with a longer response time (1 minute rather than a few seconds). TDLS systems demonstrate good gas selectivity and signal-to-noise ratios, but are relatively expensive and complex to manufacture.

${ }^{*}$ j.hodgkinson@ cranfield.ac.uk

Optical Sensing and Detection III, edited by Francis Berghmans, Anna G. Mignani,

Piet De Moor, Proc. of SPIE Vol. 9141, 91410K · (C) 2014 SPIE

CCC code: $0277-786 \mathrm{X} / 14 / \$ 18 \cdot$ doi: $10.1117 / 12.2052460$

Proc. of SPIE Vol. $914191410 \mathrm{~K}-1$ 
Here, we assess the potential for formaldehyde measurement using a non-dispersive ultra-violet technique, taking advantage of the chemical's absorption profile in the UV and new developments in UV light emitting diodes (LEDs). Part of our motivation derives from the high degree of uptake of non-dispersive infra-red (NDIR) sensors in real applications, owing to their relative simplicity and, using the strong infra-red absorption of certain gases such as carbon dioxide, compact size ${ }^{[9]}$. Such sensors are stable, field-proven, and show negligible cross-sensitivity to other species as long as the absorption bands used are chosen with care.

\section{NON-DISPERSIVE ULTRA-VIOLET SPECTROSCOPY}

Optical gas detection using absorption spectroscopy is based on application of the Beer Lambert Law ${ }^{[10]}$;

$$
I=I_{0} \exp (-\alpha \ell)
$$

Where $I$ is the light transmitted through the gas cell, $I_{0}$ is the light incident on the gas cell, $\alpha$ is the absorption coefficient of the sample (typically with units of $\mathrm{cm}^{-1}$ ) and $\ell$ is the cell's optical pathlength (typically with units of $\mathrm{cm}$ ). $\alpha$ is the product of the gas concentration (for example in atm - the partial pressure in atmospheres) and the specific absorptivity of the gas $\varepsilon$ (for example in $\mathrm{cm}^{-1} \mathrm{~atm}^{-1}$ ). The dimensionless quantity $\alpha \ell$ is known as the absorbance, in units known as absorbance units (AU). The UV absorption spectrum of formaldehyde is shown in Figure 1.

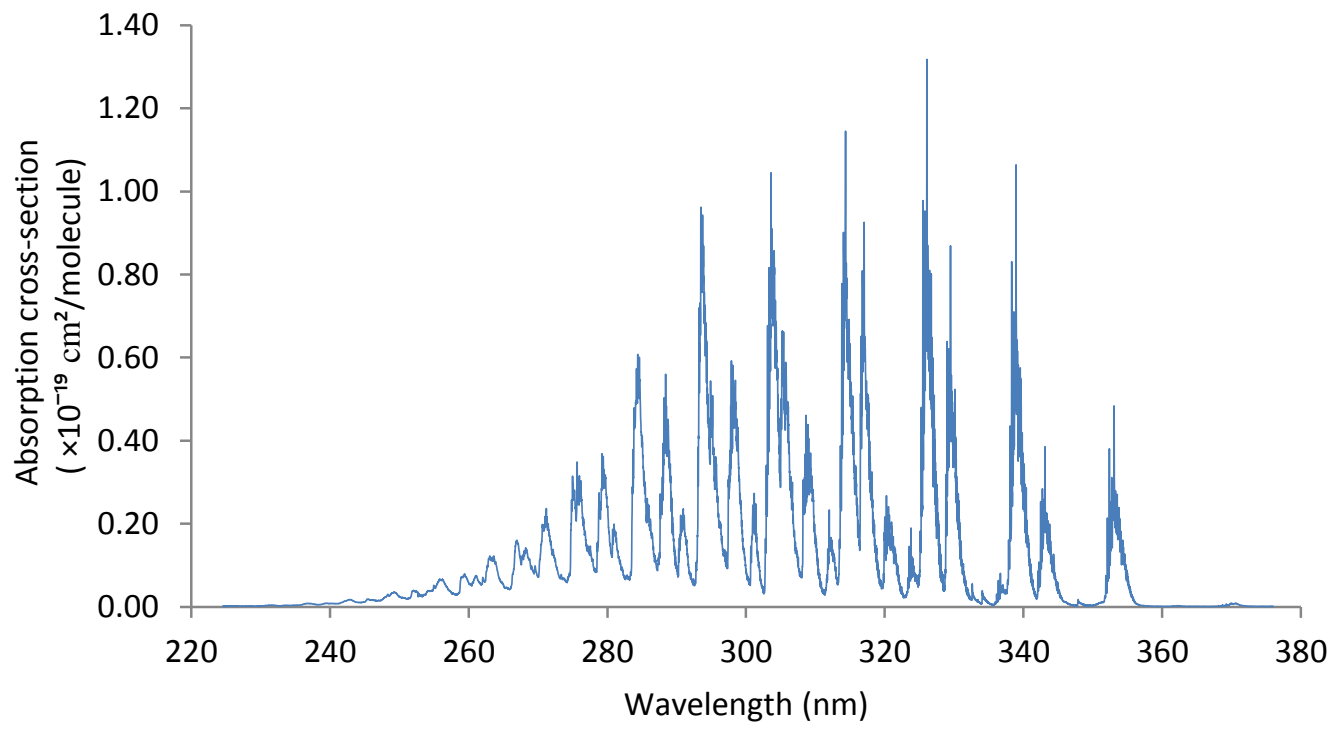

Figure 1. Graph of the formaldehyde UV absorption spectrum, re-plotted using data from Meller and Moortgat ${ }^{[11]}$.

In non-dispersive spectroscopy, light from a broadband source (in this case, a UV LED) is transmitted through a sample and optically filtered using an interference filter matched to an absorption band of the target gas (the active channel). A second reference channel, using a filter matched to a region of negligible absorption, compensates for changes in the intensity of the light source. This principle is illustrated for formaldehyde in the $340 \mathrm{~nm}$ region in Figure 2.

Transmission through the active channel comprises the integrated gas absorption:

$$
I=I_{0} \int E_{S}(\lambda) T_{A}(\lambda) \exp [-\alpha(\lambda) \ell] \mathrm{d} \lambda
$$

Where $E_{s}$ is the emission envelope of the source and $T_{A}$ is the transmission of the active channel filter. For low $\alpha \ell$, equation (2) is linear with $\alpha$, and therefore with gas concentration. In the region of linear operation, we can adapt equation (2) to give; 


$$
\alpha^{\prime} \ell=1-\frac{I_{A}}{I_{0 A}}
$$

where the subscript A denotes the active channel, and $\alpha^{\prime}$ is a pseudo absorption coefficient that follows from equation (2). Assuming that, in the absence of gas, the light transmitted through the reference channel is proportional to that transmitted through the active channel, we have;

$$
\alpha^{\prime} \ell=1-\frac{I_{O R}}{I_{0 A}} \frac{I_{A}}{I_{R}}
$$

Where the subscript R denotes the reference channel. The value of $I_{0 \mathrm{R}} / I_{0 \mathrm{~A}}$ may be determined by flushing the sample cell with clean air.

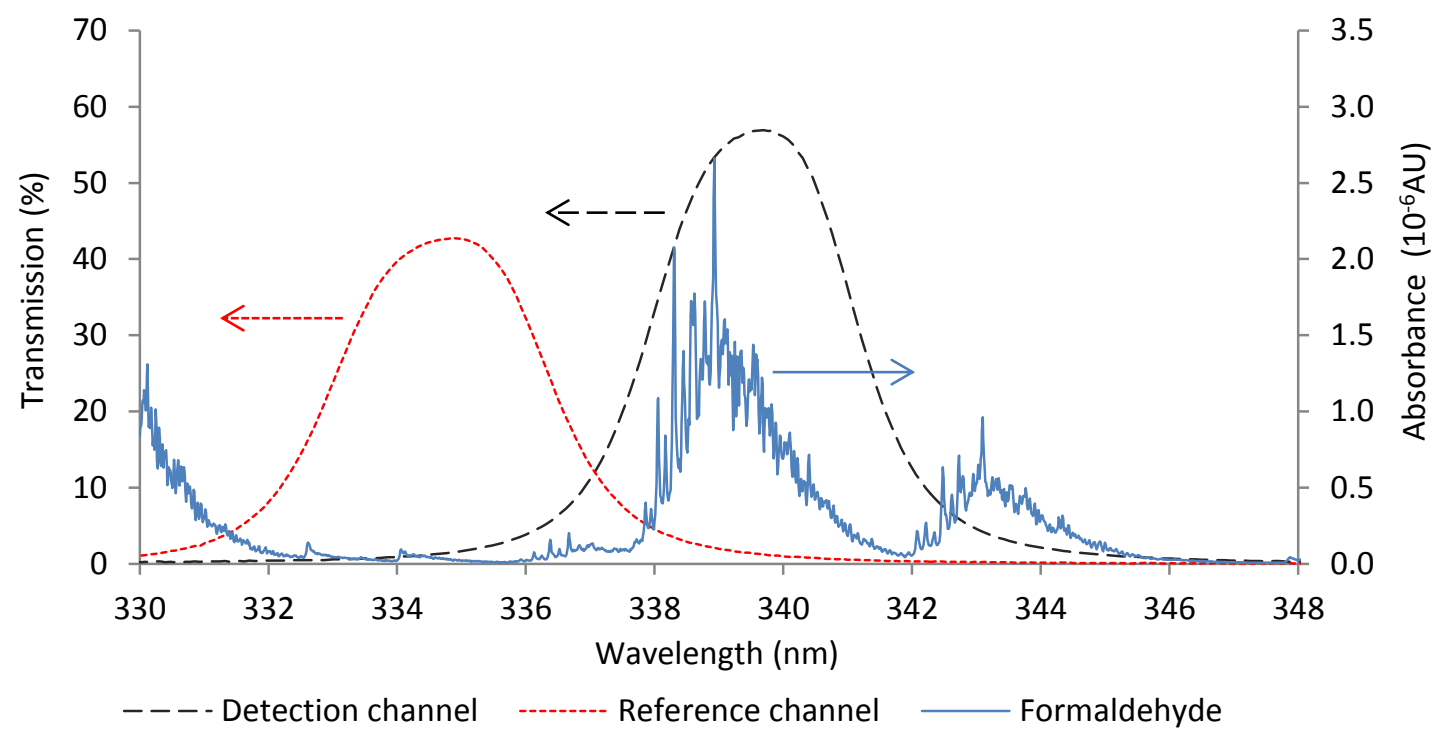

Figure 2. Intensity spectra of the detection and reference channels of the two filter system. The absorbance spectrum for $10 \mathrm{ppb}$ of formaldehyde with a $100 \mathrm{~mm}$ path length was calculated from data from Meller and Moortgat ${ }^{[11]}$.

\section{EXPERIMENTAL}

A diagram of the optical setup is shown in Figure 7.3. Light from a 340nm UV LED was collimated into a $195 \mathrm{~mm}$ long gas cell, and divided into two detection channels using a beamsplitter. Light from each channel then passed through a narrowband filter and focused onto a photodiode for detection.

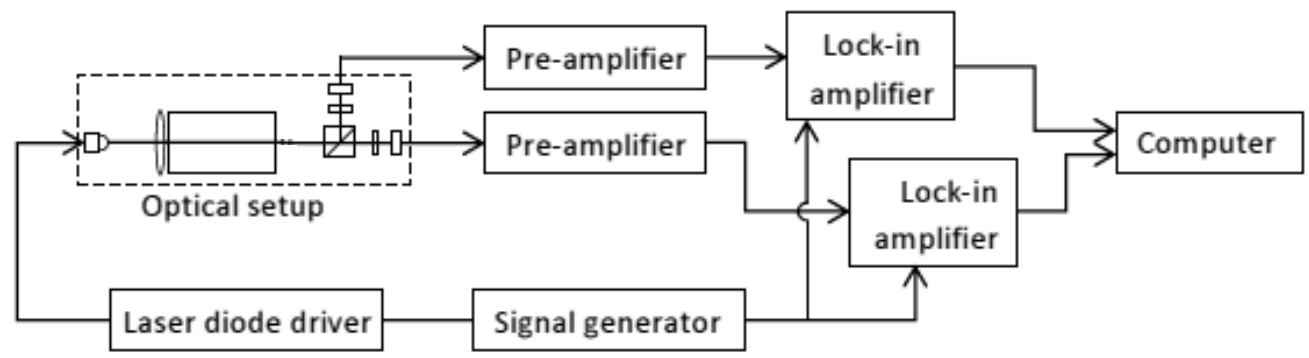

Figure 3. Configuration for formaldehyde detection using a two channel non-dispersive ultra-violet measurement. 
The LED (SETi UVTOP335TO39BL) had a central wavelength of $340 \mathrm{~nm}$ and an FWHM of 9 nm. It came with an internal parabolic mirror and a front ball lens with a $3.18 \mathrm{~mm}$ radius. The LED was driven using a signal generator (Hewlett-Packard 33120a) combined with a low noise laser diode driver (Thorlabs LDC200CV) to give a square wave modulated current, set to $9.41 \mathrm{~mA} \mathrm{p}$-p at a frequency of $6.5 \mathrm{kHz}$. Temperature was initially controlled by an externally mounted Peltier module and thermistor, but it was found that this did not give sufficient temperature stability. Therefore the system was placed in a sealed room with a stable temperature, and allowed to reach thermal equilibrium.

Two custom built laser-line filters were obtained, with a central wavelength of 339nm, a FWHM of $3.5 \mathrm{~nm}$ and a peak transmission of $51 \%$. These were angled at $0^{\circ}$ to the optic axis for the detection channel and $14^{\circ}$ for the reference channel, giving the transmission spectra shown in Figure 2. After passing through the filter, the collimated beam in each channel was focused onto a $5.8 \mathrm{~mm}^{2}$, UV-enhanced silicon photodiode (RS Components OSD5.8-7Q). The signal from the photodiodes was amplified by variable gain transimpedance amplifiers (FEMTO DLPCA-200). Signals were then input to a matched pair of lock-in amplifiers (Stanford SR850, time constant $10 \mathrm{ms).} \mathrm{Their} \mathrm{outputs} \mathrm{were} \mathrm{fed} \mathrm{to} \mathrm{a}$ computer-based data acquisition system implemented in Labview, which performed further signal averaging over a total measurement integration period of $20 \mathrm{~s}$, and calculated the absorbance according to equation (4).

\section{RESULTS}

The two filter system was tested using formaldehyde from a vapour generator (Owlstone OVG-4) using a calibrated permeation tube filled with para-formaldehyde. The permeation tube yielded formaldehyde gas when heated to $100^{\circ} \mathrm{C}$, which could be mixed with clean air to vary the concentration. Initially the air flow was switched between 500 and $50 \mathrm{~cm}^{3} \mathrm{~min}^{-1}$ to give concentration step changes between 44 and $4.4 \mathrm{ppm}$, giving a step difference of $40 \pm 7 \mathrm{ppm}$. The air flow was switched over multiple times during, in the manner of a modulated signal. The results are shown in Figure 7.27. Using this data, the short-term limit of detection was estimated to be approximately 6.6 ppm, corresponding to a minimum detectable absorbance of $4.5 \times 10^{-5} \mathrm{AU}\left(\right.$ as $\left.\Delta \mathrm{I} / \mathrm{I}_{0}\right)$.

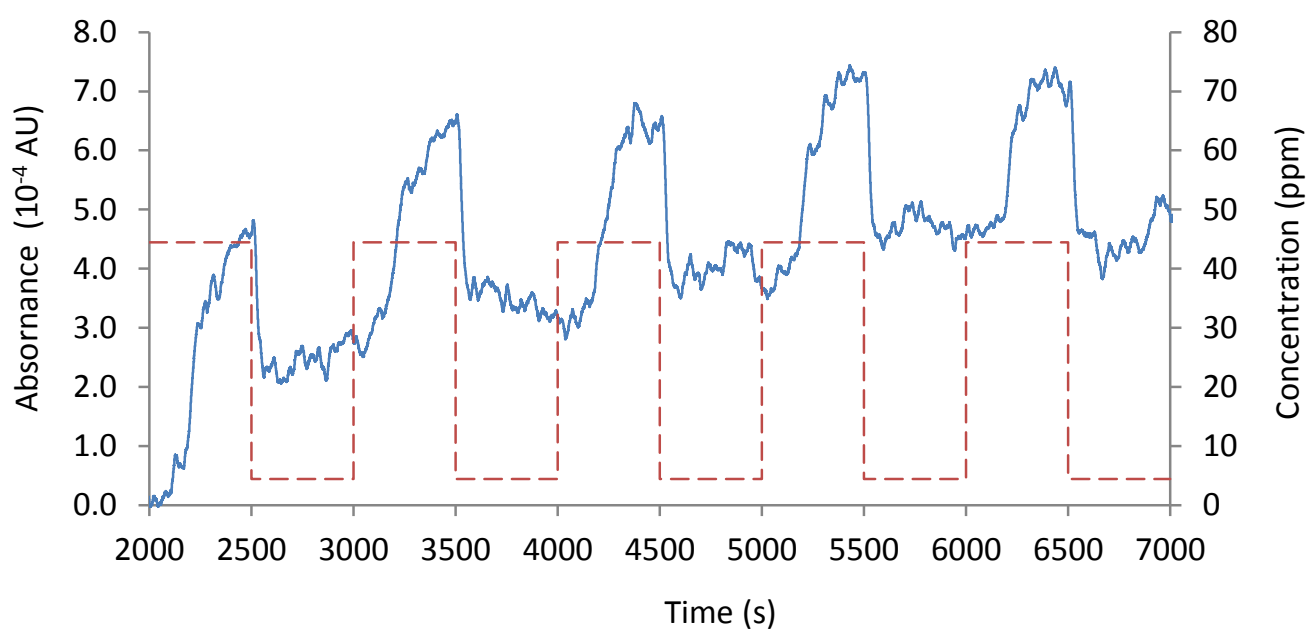

Figure 4. Graph of absorbance measured by the system (solid line) caused by a periodic steps in formaldehyde concentration between 4.4ppm and 44ppm (dashed line). A long-term drift in the baseline can be seen.

Secondly, the air flow through the vapour generator was altered over the range $500-50 \mathrm{~cm}^{3} \mathrm{~min}^{-1}$ to produce a calibration plot. We found that over longer time periods, baseline drifts (visible in Figure 4) caused the limit of detection to deteriorate to $30 \mathrm{ppm}$ or $2 \times 10^{4} \mathrm{AU}$, as shown in Figure 5 . 


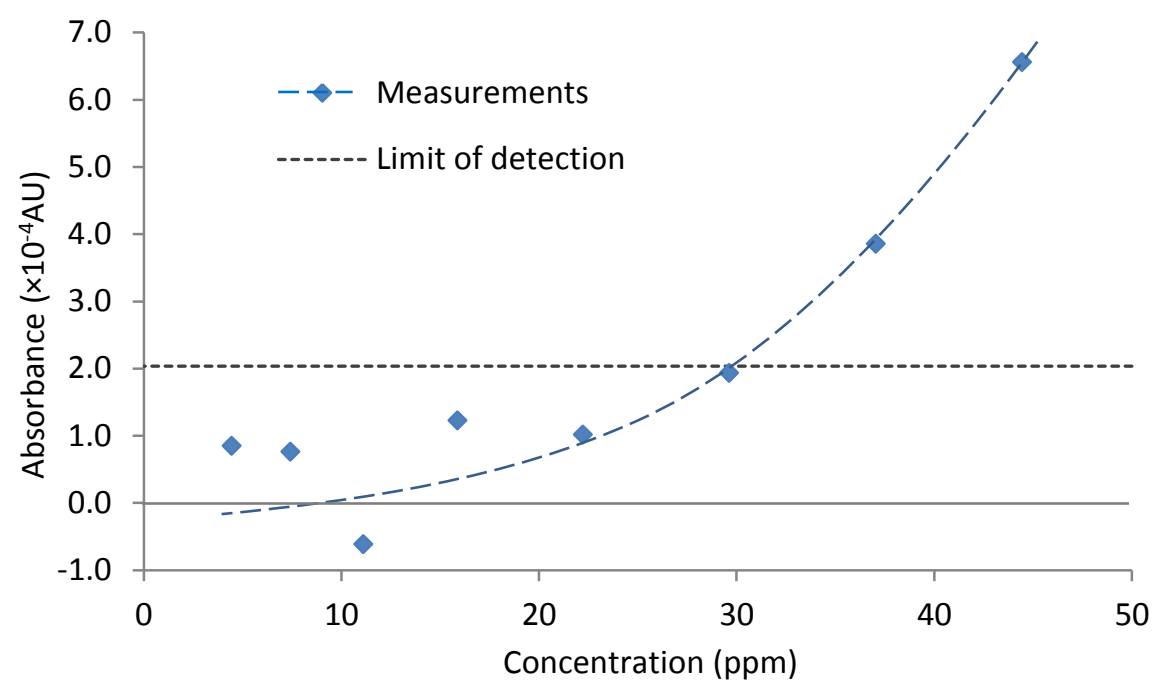

Figure 5. Calibration plot for absorbance measurement (as $\Delta \mathrm{I} / \mathrm{I}_{0}$ ) versus formaldehyde concentration, showing the long-term limit of detection. The latter was dominated by unwanted, thermally induced spectral drifts in the LED output.

\section{DISCUSSION AND CONCLUSIONS}

Our tests have shown an estimated short-term (20s) limit of detection for formaldehyde of $6.6 \mathrm{ppm}$, corresponding to an absorbance of $4.5 \times 10^{-5} \mathrm{AU}$, which for over longer time periods (several minutes) deteriorated to $30 \mathrm{ppm}$ or $2 \times 10^{4} \mathrm{AU}$. Clearly, this is not good enough to detect formaldehyde at the WHO recommended limit of $80 \mathrm{ppb}$, therefore it is worth discussing whether this performance could be improved.

Our long-term performance was limited by unwanted spectral variation of the LED with temperature, which affected the active and reference channels disproportionately, leading to spurious absorption measurements (either positive or negative). Several studies have shown a variation in LED output with temperature ${ }^{[12,13,14]}$, finding a decrease in intensity with increasing temperature as well as a shift in peak wavelength. If the temperature control of the LED could be improved (for example, by using a Peltier and thermistor internal to the device package, as it the case with many infrared laser diodes), there is potential to reduce the limit of detection. For a system using this LED, with a specified typical emission of $400 \mu \mathrm{W}$, the ultimate performance would be dictated by detector noise at a level corresponding to $2.4 \times 10^{-5} \mathrm{AU}$ or $3.5 \mathrm{ppm}$. At this level, an increase in optical pathlength from $195 \mathrm{~mm}$ to approximately $7 \mathrm{~m}$ would be required to give a limit of detection of $100 \mathrm{ppb}$. Even using folded optical paths, this would be difficult to achieve in a compact, eg hand-portable instrument, suggesting that a higher source intensity would be needed. We therefore conclude that improvements in both UV LED output intensity and thermal control are required before they can be used in this manner for measurements at the action limits for indoor formaldehyde concentration.

To conclude, we have developed a formaldehyde sensor based on a non-dispersive ultra-violet measurement principle that makes use of the absorption band at $340 \mathrm{~nm}$ and recent advances in UV LEDs, for the first time. A short-term limit of detection of $6.6 \mathrm{ppm}$ was estimated. This deteriorated to $30 \mathrm{ppm}$ for longer term measurement made over several minutes, owing to thermally-induced spectral change in the LED's output over time. The work demonstrates that this approach can be used in principle, and points towards a need for UV LEDs with higher output intensity and superior thermal control.

\section{ACKNOWLEDGEMENTS}

This project was part funded by the EPSRC (grants EP/P504880 and EP/H02252X). 


\section{REFERENCES}

[1] Cross, J. H., Limero, T. F., Lane, J. L. and Wang, F. "Determination of ammonia in ethylene using ion mobility spectrometry." Talanta 45, 19-23 (1997).

[2] Lu, Y., Liu, J., Lu, B,. Jiang, A. and Wan, C. "Study on the removal of indoor VOCs using microtechnology." J. Hazard. Mater. 182, 204-209 (2010).

[3] Nielsen, G. D. and Wolkoff, P. "Cancer effects of formaldehyde: a proposal for an indoor air guideline value." Arch. Toxicol. 84, 423-446 (2010).

[4] Kim, S. "Control of formaldehyde and TVOC emission from wood-based flooring composites at various manufacturing processes by surface finishing." J. Hazard. Mater. 176, 14-19 (2010).

[5] Kudo, H., Suzuki, Y., Gessei, T., Takahashi, D., Arakawa, T. and Mitsubayashi, K. "Biochemical gas sensor (biosniffer) for ultrahigh-sensitive gaseous formaldehyde monitoring." Biosens. Bioelecton. 26, 854-858 (2010).

[6] P.F h.3.P.I. Monitor Operation Manual, PPM Technology Ltd., Caernarfon, UK (2008).

[7] Rehle, D., Leleux, D., Erdelyi, M., Tittel, F., Fraser, M. and Friedfeld, S. "Ambient formaldehyde detection with a laser spectrometer based on difference-frequency generation in PPLN." Appl. Phys. B 72, 947-952 (2001).

[8] Richter, D. Fried, A. Wert, B. P. Walega, J. G. and Tittel, F. K. "Development of a tunable mid-IR difference frequency laser source for highly sensitive airborne trace gas detection." Appl. Phys. B 75, 281-288 (2002).

[9] Hodgkinson, J., Smith, R., Ho, W. O., Saffell, J. R. and Tatam, R. P. "Non-dispersive infra-red (NDIR) measurement of carbon dioxide at $4.2 \mu \mathrm{m}$ in a compact and optically efficient sensor." Sensor. Actuat. B 186, 580588 (2013).

[10] Ingle, J.D. and Crouch, S.R.. [Spectrochemical Analysis]. Prentice Hall, London (1988).

[11] Meller, R. and Moortgat, G. K. "Temperature dependence of the absorption cross sections of formaldehyde between 223 and $323 \mathrm{~K}$ in the wavelength range 225-375 nm," J. Geophys. Res.-Atmos. 105, 7089-7101 (2000).

[12] Xi, Y., Xi, J. Q., Gessmann, T., Shah, J. M., Kim, J. K., Schubert, E. F., Fischer, A. J., Crawford, M. H., Bogart, K. H. A. and Allerman, A. A. "Junction and carrier temperature measurements in deep-ultraviolet light-emitting diodes using three different methods." Appl. Phys. Lett. 86, 031907 (2005).

[13] Reynolds, K. J., Dekock, J. P., Tarassenko, L. and Moyle, J. T. B. "Temperature dependence of LED and its theoretical effect on pulse oximetry," Brit. J. Anaesth. 67 (5), 638-643 (1991).

[14] Wang, J. C., Fang, C. H., Wu, Y. F., Chen, W. J., Kuo, D. C. and Fan, P. L. "The effect of junction temperature on the optoelectrical properties of InGaN/GaN multiple quantum well light-emitting diode." J. Lumin. 132, 429433 (2012). 